\title{
A closed formula for the number of convex permutominoes
}

\author{
Filippo Disanto * $\quad$ Andrea Frosini ${ }^{\dagger} \quad$ Renzo Pinzani ${ }^{\dagger}$ \\ Simone Rinaldi*
}

Submitted: Feb 23, 2007; Accepted: Jul 28, 2007; Published: Aug 20, 2007

Mathematical Subject Classification: 05A15

\begin{abstract}
In this paper we determine a closed formula for the number of convex permutominoes of size $n$. We reach this goal by providing a recursive generation of all convex permutominoes of size $n+1$ from the objects of size $n$, according to the ECO method, and then translating this construction into a system of functional equations satisfied by the generating function of convex permutominoes. As a consequence we easily obtain also the enumeration of some classes of convex polyominoes, including stack and directed convex permutominoes.
\end{abstract}

\section{Basic definitions and contents of the paper}

A polyomino is a finite union of elementary cells of the lattice $Z \times Z$, whose interior is connected (see Figure $1(a)$ ). Polyominoes are defined up to a translation. A polyomino is said to be column convex (resp. row convex) if all its columns (resp. rows) are connected (see Figure $1(b)$ ). A polyomino is said to be convex, if it is both row and column convex (see Figure $1(c)$ ).

Delest and Viennot [13] determined the number $c_{n}$ of convex polyominoes with semiperimeter $n+2$,

$$
c_{n+2}=(2 n+11) 4^{n}-4(2 n+1)\left(\begin{array}{c}
2 n \\
n
\end{array}\right), \quad n \geq 0 ; \quad c_{0}=1, \quad c_{1}=2,
$$

sequence $A 005436$ in [18], the first few terms being:

$$
1,2,7,28,120,528,2344,10416, \ldots
$$

*Università di Siena, Dipartimento di Scienze Matematiche e Informatiche, Pian dei Mantellini 44, 53100 Siena, Italy (rinaldi@unisi.it).

${ }^{\dagger}$ Università di Firenze, Dipartimento di Sistemi e Informatica, viale Morgagni 65, 50134 Firenze, Italy ([frosini, pinzani]@dsi.unifi.it). 


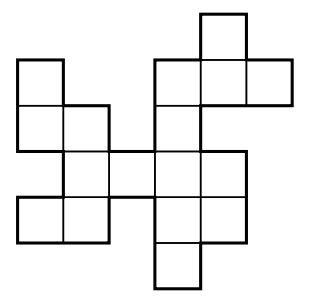

(a)

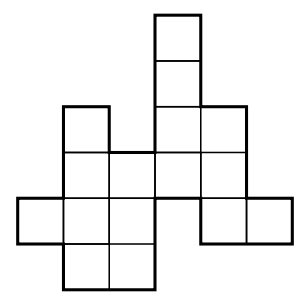

(b)

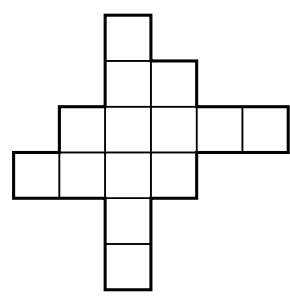

(c)

Figure 1: (a) a polyomino; (b) a column convex polyomino; (c) a convex polyomino.

In the last two decades convex polyominoes, and several combinatorial objects obtained as a generalizations of this class, have been studied by various points of view. For the main results concerning the enumeration and other combinatorial properties of convex polyominoes we refer to $[6,7,8,10]$.

\section{$1.1 \quad$ Permutominoes}

Let $P$ be a polyomino, having $n$ rows and columns, $n \geq 1$; we assume without loss of generality that the south-west corner of its minimal bounding rectangle is placed in $(1,1)$. Let $\mathcal{A}=\left\{A_{1}, \ldots, A_{2(r+1)}\right\}$ be the set of its vertices ordered in a clockwise sense starting from the leftmost vertex having minimal ordinate.

We say that $P$ is a permutomino if the sets $\mathcal{P}_{1}=\left\{A_{1}, A_{3}, \ldots, A_{2 r+1}\right\}$ and $\mathcal{P}_{2}=$ $\left\{A_{2}, A_{4}, \ldots, A_{2 r+2}\right\}$ represent two permutation matrices of $[n+1]=\{1,2, \ldots, n+1\}$. Obviously, if $P$ is a permutomino, then $r=n$, and $n$ is called the size of the permutomino.

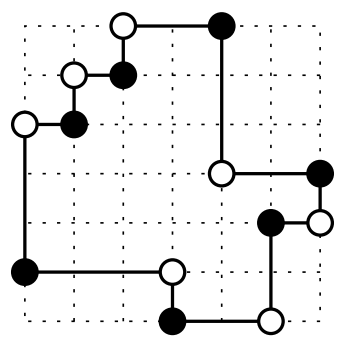

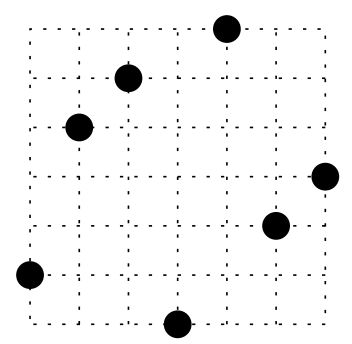

$\pi_{1}=(2,5,6,1,7,3,4)$

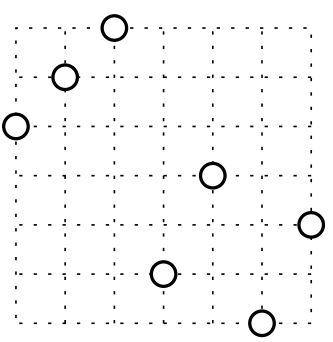

$\pi_{2}=(5,6,7,2,4,1,3)$

Figure 2: A permutomino and the two associated permutations.

The two permutations associated with $\mathcal{P}_{1}$ and $\mathcal{P}_{2}$ are indicated by $\pi_{1}$ and $\pi_{2}$, respectively (see Figure 2). While it is clear that any permutomino of size $n$ uniquely individuates two distinct permutations $\pi_{1}$ and $\pi_{2}$ of $[n+1]$, such that

i) $\pi_{1}(i) \neq \pi_{2}(i), 1 \leq i \leq n+1$ 
ii) $\pi_{1}(1)<\pi_{2}(1)$, and $\pi_{1}(n+1)>\pi_{2}(n+1)$,

not all the pairs of permutations $\left(\pi_{1}, \pi_{2}\right)$ of $n+1$ satisfying i) and ii) define a permutomino; Figure 3 depicts the two problems which may occur.

From the definition we have that in any permutomino $P$, for each abscissa (ordinate) there is exactly one vertical (horizontal) side in the boundary of $P$ with that coordinate. It is simple to observe that the previous property is also a sufficient condition for a polyomino to be a permutomino.

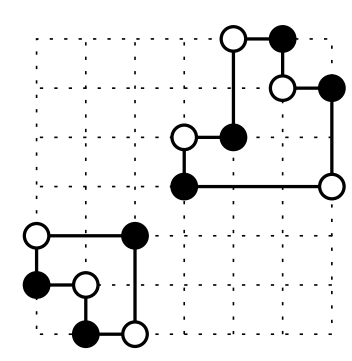

$$
\pi_{1}=(2,1,3,4,5,7,6)
$$$$
\pi_{2}=(3,2,1,5,7,6,4)
$$

(a)

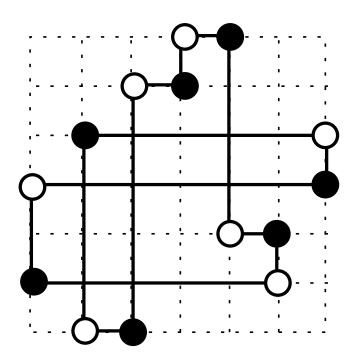

$\pi_{1}=(2,5,1,6,7,3,4)$

$\pi_{2}=(4,1,6,7,3,2,5)$

(b)

Figure 3: The two main cases when a pair of permutations $\pi_{1}$ and $\pi_{2}$ of $[n+1]$ may not define a permutomino: (a) two disconnected sets of cells; (b) the boundary crosses itself.

Permutominoes were introduced by F. Incitti in [17] while studying the problem of determining the $\widetilde{R}$-polynomials (related with the Kazhdan-Lusztig R-polynomials) associated with a pair $\left(\pi_{1}, \pi_{2}\right)$ of permutations. Concerning the class of polyominoes, our definition (though different) turns out to be equivalent to Incitti's one, which is more general but uses some algebraic notions not necessary in this paper.

In [15], using bijective techniques, it was proved that the number of parallelogram permutominoes of size $n$ is equal to the $n$th Catalan number,

$$
\frac{1}{n+1}\left(\begin{array}{c}
2 n \\
n
\end{array}\right),
$$

and moreover, that the number of directed-convex permutominoes of size $n$ is equal to half the $n$th central binomial coefficient,

$$
\frac{1}{2}\left(\begin{array}{c}
2 n \\
n
\end{array}\right) .
$$

In this paper we deal with the enumeration of convex polyominoes which are also permutominoes, the so called convex permutominoes. We reach this goal by determining 
a direct recursive construction for the convex permutominoes of a given size, based on the application of the ECO method, which easily leads to the generating function, and finally prove that the number of convex permutominoes of size $n$ is:

$$
f_{n}=2(n+3) 4^{n-2}-\frac{n}{2}\left(\begin{array}{c}
2 n \\
n
\end{array}\right) \quad n \geq 1 .
$$

We point out that the same enumerative result has been recently obtained, independently, and with different techniques, by Boldi et al. [5].

\subsection{ECO method}

In this section we will recall some basics about the ECO method, where ECO stands for Enumeration of Combinatorial Objects. Such a method, introduced by Pinzani and his collaborators in [3], is a constructive method to produce all the objects of a given class, according to the growth of a certain parameter (the size) of the objects. Basically, the idea is to perform "local expansions" on each object of size $n$, thus constructing a set of objects of the successive size (see [3] for more details).

The application of the ECO method often leads to an easy solution for problems that are commonly believed "hard" to solve. For example, in [14] the authors give an ECO construction for the classes of convex polyominoes and column-convex polyominoes according to the semi-perimeter. A simple algebraic computation leads then to the determination of generating functions for the two classes.

In [1] it is also shown that an ECO construction easily leads to an efficient algorithm for the exhaustive generation of the examined class. Moreover, an ECO construction can often produce interesting combinatorial information about the class of objects studied, as shown in [3] using analytic methods, or in [4], using bijective techniques. In [2], Banderier et al. reintroduced the kernel method in order to determine the generating function of various types of ECO systems.

Going deeper into formalism, let $p$ be a parameter $p: \mathcal{O} \rightarrow \mathbb{N}^{+}$, such that $\left|\mathcal{O}_{n}\right|=$ $|\{O \in \mathcal{O}: p(O)=n\}|$ is finite. An operator $\vartheta$ on the class $\mathcal{O}$ is a function from $\mathcal{O}_{n}$ to $2^{\mathcal{O}_{n+1}}$, where $2^{\mathcal{O}_{n+1}}$ is the power set of $\mathcal{O}_{n+1}$.

Proposition 1 Let $\vartheta$ be an operator on $\mathcal{O}$. If $\vartheta$ satisfies the following conditions:

1. for each $O^{\prime} \in \mathcal{O}_{n+1}$, there exists $O \in \mathcal{O}_{n}$ such that $O^{\prime} \in \vartheta(O)$,

2. for each $O, O^{\prime} \in \mathcal{O}_{n}$ such that $O \neq O^{\prime}$, then $\vartheta(O) \cap \vartheta\left(O^{\prime}\right)=\emptyset$,

then the family of sets $\left\{\vartheta(O): O \in \mathcal{O}_{n}\right\}$ is a partition of $\mathcal{O}_{n+1}$.

This method was successfully applied to the enumeration of various classes of walks, permutations, and polyominoes. We refer to [3], and [16] for further details and results.

The recursive construction determined by $\vartheta$ can be suitably described through a generating tree, i.e. a rooted tree whose vertices are objects of $\mathcal{O}$. The objects having the 
same value of the parameter $p$ lie at the same level, and the sons of an object are the objects it produces through $\vartheta$.

If the construction determined by the ECO operator $\vartheta$ is regular enough it is then possible to describe it by means of a succession rule of the form:

$$
\left\{\begin{array}{l}
(b) \\
(d) \rightsquigarrow\left(c_{1}\right)\left(c_{2}\right) \ldots\left(c_{q(d)}\right),
\end{array}\right.
$$

where $b, d, c_{i} \in \mathbb{N}$, and $q: \mathbb{N}^{+} \rightarrow \mathbb{N}^{+}$. To each object $O$ in the generating tree of $\vartheta$ is associated a degree $d(O)$ (briefly, $d$ ) which explains how many objects are produced by

$O$ through $\vartheta$. In practice, the succession rule (3) reads that the object at the root of the generating tree has degree $b$, and every object $O$ with degree $d$ in the generating tree has $q(d)$ objects $O_{1}^{\prime}, \ldots, O_{q(d)}^{\prime}$, where each $O_{i}$ has degree $c_{i}, 1 \leq i \leq q(d)$. A succession rule defines a sequence $\left\{f_{n}\right\}_{n \geq 1}$ of positive integers, where $f_{n}$ is the number of nodes at level $n$ of the generating tree, assuming that the root is at level 1.

\section{Generation of convex permutominoes}

Let $\mathcal{C}_{n}$ be the set of convex permutominoes of size $n$. In order to define the ECO construction for convex permutominoes, we need to point out a simple property of their boundary, related to reentrant and salient points. So let us briefly recall the definition of these objects.

Let $P$ be a polyomino; starting from the leftmost point having minimal ordinate, and moving in a clockwise sense, the boundary of $P$ can be encoded as a word in a four letter alphabet, $\{N, E, S, W\}$, where $N$ (resp. $E, S, W$ ) represents a north (resp.east, south, west) unit step. Any occurrence of a sequence $N E, E S, S W$, or $W N$ in the word encoding $P$ defines a salient point of $P$, while any occurrence of a sequence $E N, S E, W S$, or $N W$ defines a reentrant point of $P$ (see for instance, Figure 4).

Reentrant and salient points were considered in [12], and successively in [9], in a more general context, and it was proved that in any polyomino the difference between the number of salient and reentrant points is equal to 4 .

Let us turn to consider the class of convex permutominoes. In a convex permutomino of size $n$ the length of the word coding the boundary is $4 n$, and we have $n+3$ salient points and $n-1$ reentrant points; moreover we observe that a reentrant point cannot lie on the minimal bounding rectangle. This leads to the following remarkable property:

Proposition 2 The set of reentrant points of a convex permutomino of size $n$ defines a permutation matrix of $[n-1], n \geq 2$.

For simplicity of notation, and to clarify the definition of the upcoming ECO construction, we agree to group the reentrant points of a convex permutomino in four classes; namely, we choose to represent a reentrant point determined by a sequence $E N$ (resp. $S E, W S, N W)$ with the symbol $\alpha($ resp. $\beta, \gamma, \delta)$. Using this notation we can state that 


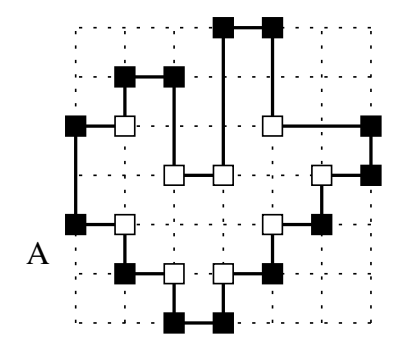

NNENESSENNNESSEESWSWSWSWNWNW

Figure 4: The coding of the boundary of a polyomino, starting from $A$ and moving in a clockwise sense; its salient (resp. reentrant) points have been evidenced by a black (resp. white) square.

each convex permutomino of size $n \geq 2$ can be uniquely represented by the permutation matrix defined by its reentrant points, which has dimension $n-1$, and uses the symbols $\alpha, \beta, \gamma, \delta$.

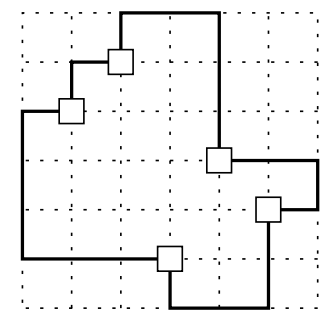

$\left[\begin{array}{ccccc}0 & \alpha & 0 & 0 & 0 \\ \alpha & 0 & 0 & 0 & 0 \\ 0 & 0 & 0 & \beta & 0 \\ 0 & 0 & 0 & 0 & \gamma \\ 0 & 0 & \delta & 0 & 0\end{array}\right]$

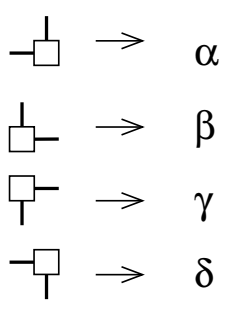

Figure 5: The reentrant points of a convex permutomino uniquely define a permutation matrix in the symbols $\alpha, \beta, \gamma$ and $\delta$.

\subsection{The ECO operator}

Let $P \in \mathcal{C}_{n}$; the number of cells in the rightmost column of $P$ is called the degree of $P$. For any $n \geq 1$ we partition the class $\mathcal{C}_{n}$ into three distinct classes. In order to define these classes, let us consider the following conditions on a convex permutomino:

U1 : the uppermost cell of the rightmost column of the polyomino has the maximal ordinate among all the cells of the polyomino;

U2 : the lowest cell of the rightmost column of the polyomino has the minimal ordinate among all the cells of the polyomino.

We say that a convex permutomino $P$ belongs to class: 


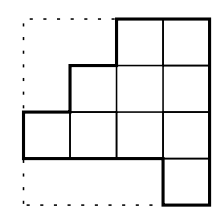

(B)

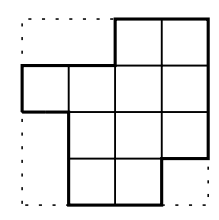

$(\mathrm{R})$

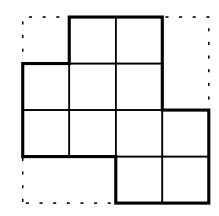

(R)

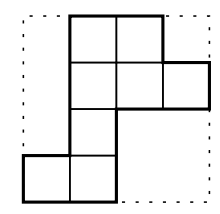

(G)

Figure 6: Convex permutominoes in classes $B, R$, and $G$.

- $B$, if it satisfies both conditions $\mathbf{U} 1$ and U2 (i.e. $P$ has degree $n$, see Figure 6, (B)); we observe that the single cell permutomino belongs to class $B$.

- $R$, if it satisfies only one among conditions U1, U2 (see Figure 6, (R));

- $G$, if it satisfies none of conditions U1, U2 (see Figure 6, (G)).

For simplicity sake, each permutomino in class $B$ (resp. $R, G$ ) and degree $k$ is represented by the label $(k)_{b}$ (resp. $\left.(k)_{r},(k)_{g}\right)$. For instance, the four permutominoes depicted in Figure 6 have labels $(4)_{b},(3)_{r},(2)_{r},(1)_{g}$, respectively. In particular the single cell permutomino has the label $(1)_{b}$.

Our aim is now to use the property stated in Proposition 2 to define an ECO operator $\vartheta: \mathcal{C}_{n} \rightarrow 2^{\mathcal{C}_{n+1}}$ which defines a recursive construction of all the convex permutominoes of size $n+1$ in a unique way from the objects of size $n$. The operator $\vartheta$ acts on a convex permutomino performing some local expansions on the cells of its rightmost column. In order to define these operations let us consider a generic permutomino $P$ of size $n$, let us indicate by $c_{1}, \ldots, c_{n}$ (resp. $r_{1}, \ldots, r_{n}$ ) the columns (resp. rows) of $P$ numbered from left to right (resp. bottom to top), and by $\ell\left(c_{i}\right)$ (resp. $\ell\left(r_{i}\right)$ ) the number of cells in the $i$ th column (resp. $i$ th row), with $1 \leq i \leq n$. The four operations of $\vartheta$ will be denoted by $\alpha$, $\beta, \gamma$, and $\delta$, and below we give a detailed description of each of them:

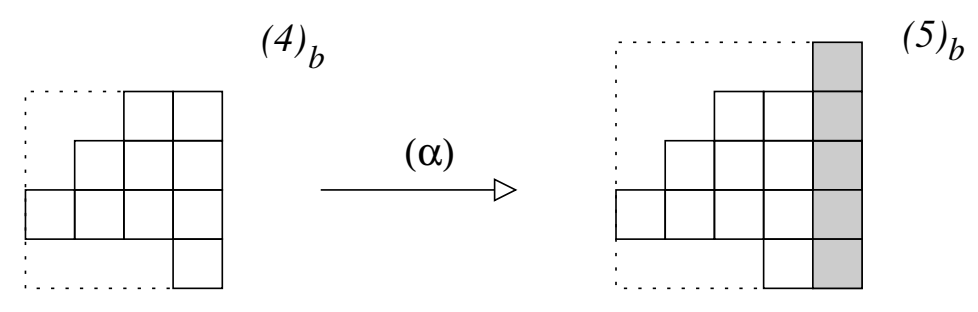

Figure 7: Operation $(\alpha)$ performed on a permutomino of class $B$. The added column has been highlighted. 
$(\alpha)$ if $P$ satisfies condition $\mathbf{U 1}$, then $(\alpha)$ adds a new column made of $c_{n}+1$ cells on the right of $c_{n}$, according to Figure 7 .

It is clear that the obtained polyomino is a convex permutomino of size $n+1$, still satisfying condition $\mathbf{U 1}$; the rightmost reentrant point in such new permutomino is of type $\alpha$ (this is the reason why we have called the reentrant points with the same name of the operations on permutominoes).

$(\beta)$ it can be performed on each cell of $c_{n}$; so let $d_{i}$ be the $i$ th cell of $c_{n}$, from bottom to top, with $1 \leq i \leq \ell\left(c_{n}\right)$. Operation $(\beta)$ adds a new row above the row containing $d_{i}$ (of the same length), and a new column on the right of $c_{n}$, made of $i$ cells, as illustrated in Figure 8.

Observe that, since the new added row is long as the row below it, we ensure that the obtained polyomino has a unique horizontal side at level $i$, while adding the new column from bottom to level $i$ we ensure that the obtained polyomino has a unique vertical side at abscissa $n-1$, hence the basic property of permutominoes is preserved.
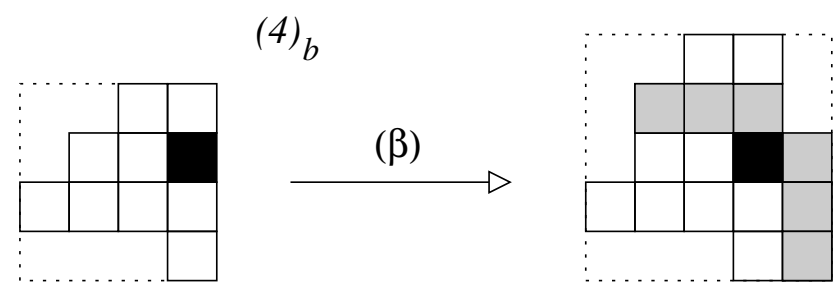

$(3)_{r}$

Figure 8: Operation $(\beta)$ performed on a cell $d_{i}$ of the rightmost column of a polyomino in class $B$. The cell $d_{i}$ is filled in black, the added row and column have been highlighted.

Then it is clear that, for any $i$, the obtained polyomino is a convex permutomino of size $n+1$, and its rightmost reentrant point is of type $\beta$.

$(\gamma)$ it can be performed on each cell of $c_{n}$; so let $d_{i}$ be the $i$ th cell of $c_{n}$, from bottom to top, with $1 \leq i \leq \ell\left(c_{i}\right)$. Operation $(\gamma)$ adds a new row below the row containing $d_{i}$ (of the same length), and a new column on the right of $c_{n}$, made of $n-i+1$ cells, as illustrated in Figure 9.

It is clear that, for any $i$, the obtained polyomino is a convex permutomino of size $n+1$, and its rightmost reentrant point is of type $\gamma$.

$(\delta)$ if $P$ satisfies condition $\mathbf{U} \mathbf{2}$, then $(\delta)$ adds a new column made of $c_{n}+1$ cells on the right of $c_{n}$, according to Figure 10 .

It is clear that the obtained polyomino is a convex permutomino of size $n+1$, still satisfying condition U2; the rightmost reentrant point in such new permutomino is of type $\delta$. 
$(4)_{b}$

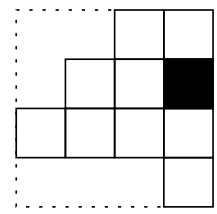

$(\gamma)$

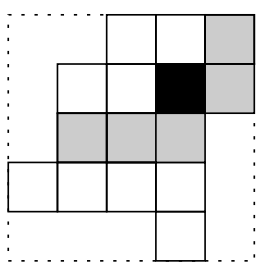

$(2)_{r}$

$(5)_{b}$

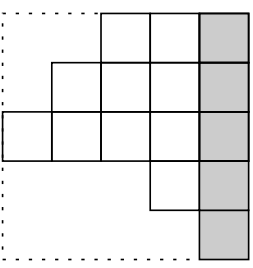

Figure 10: Operation $(\delta)$ performed a polyomino in class $B$.

As we already mentioned, the operations performed by $\vartheta$ on a convex permutomino $P$ depend on the family to which $P$ belongs. So let us consider the different cases:

1. $P$ belongs to the class $B$. The operator $\vartheta$ performs on $P$ operations $(\alpha),(\delta)$ and one application of $(\beta)$ and $(\gamma)$ for any cell in $c_{n}$. So, let $k$ be the degree of $P$, the application of $\vartheta$ to $P$ produces $2 k+2$ different convex permutominoes of size $n+1$ (see Figure 11).

More formally, applying $\vartheta$ to a convex permutomino of label $(k)_{b}$, we have $2(k+1)$ different permutominoes, two for each of the labels $(1)_{r},(2)_{r}, \ldots(k)_{r}$, and two with label $(k+1)_{b}$. This can be formalized by the production:

$$
(k)_{b} \rightsquigarrow(1)_{r}(1)_{r}(2)_{r}(2)_{r} \ldots(k)_{r}(k)_{r}(k+1)_{b}(k+1)_{b} .
$$

2. $P$ belongs to the class $R$. There are two possibilities:

i. $P$ satisfies $\mathbf{U 1}$ (and not U2). The operator $\vartheta$ performs on $P$ operation $(\alpha)$, and one application of operations $(\beta)$ and $(\gamma)$ for any cell in $c_{n}$.

ii. $P$ satisfies $\mathbf{U} \mathbf{2}$ (and not $\mathbf{U 1}$ ). The operator $\vartheta$ performs on $P$ operation $(\delta)$, and one application of operations $(\beta)$ and $(\gamma)$ for any cell in $c_{n}$ (see Figure 12).

In both cases, being $k$ be the degree of $P$, the application of $\vartheta$ to $P$ produces $2 k+1$ different convex permutominoes of size $n+1$. More formally, applying $\vartheta$ to a convex 


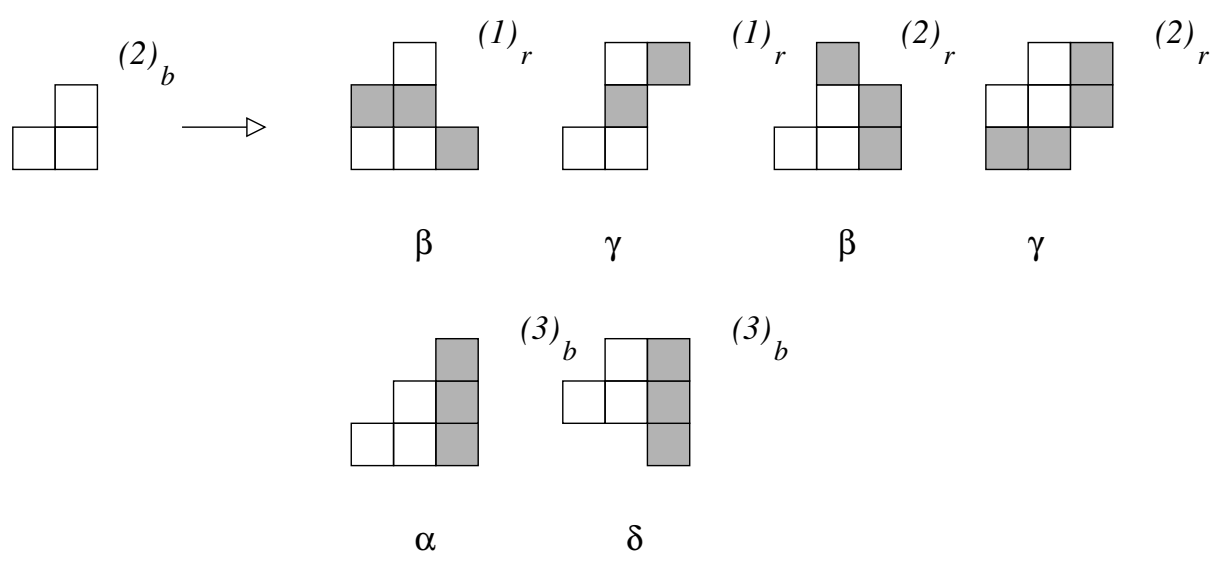

Figure 11: The operator $\vartheta$ applied to a permutomino of class $B$; the added rows and columns are highlighted, and the applied operation is mentioned below.

permutomino of label $(k)_{r}$, we have $2 k+1$ different permutominoes, for each of the labels $(1)_{r},(2)_{r}, \ldots(k)_{r},(k+1)_{r}$, and $(1)_{g},(2)_{g}, \ldots(k)_{g}$. This can be formalized by the production:

$$
(k)_{r} \rightsquigarrow(1)_{r}(1)_{g}(2)_{r}(2)_{g} \ldots(k)_{r}(k)_{g}(k+1)_{r} .
$$

3. $P$ belongs to the class $G$. The operator $\vartheta$ performs on $P$ an application of operations $(\beta)$ and $(\gamma)$ for any cell in $c_{n}$. So, let $k$ be the degree of $P$, the application of $\vartheta$ to $P$ produces $2 k$ different convex permutominoes of size $n+1$. More formally, applying $\vartheta$ to a convex permutomino of label $(k)_{g}$, we have $2 k$ different permutominoes, two for each of the labels $(1)_{g},(2)_{g}, \ldots(k)_{g}$. This can be formalized by the production:

$$
(k)_{g} \rightsquigarrow(1)_{g}(1)_{g}(2)_{g}(2)_{g} \ldots(k)_{g}(k)_{g} .
$$

Proposition 3 The operator $\vartheta$ satisfies conditions 1. and 2. of Proposition 1.

Proof. We have to prove that any convex permutomino of size $n \geq 2$ is uniquely obtained through the application of the operator $\vartheta$ to a convex permutomino of size $n-1$. So let $P \in \mathcal{C}_{n}$, and consider the rightmost reentrant point of $P$, which is unique due to Proposition 2. We have the following four possibilities:

1. the rightmost reentrant point of $P$ is of type $\alpha$, i.e. $\ell\left(c_{n}\right)=n$; due to the permutomino definition, it is clear that $\ell\left(r_{n}\right)=1$, then $P$ has been produced through the application of operation $(\alpha)$ to the permutomino $P^{\prime} \in \mathcal{C}_{n-1}$, obtained removing column $c_{n}$ from $P$ (see Figure 7 ); 


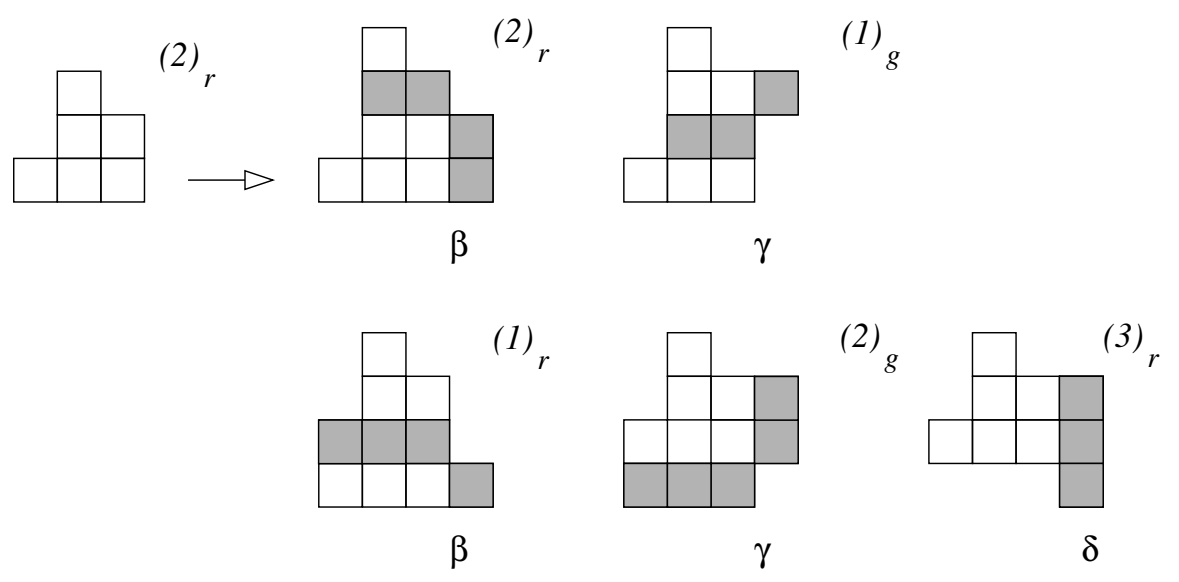

Figure 12: The operator $\vartheta$ applied to a permutomino of class $R$, satisfying $\mathbf{U} 2$ (and not U1); the added rows and columns are highlighted, and the applied operation is mentioned below.

2. the rightmost reentrant point of $P$ is of type $\beta$, and then necessarily $1 \leq \ell\left(c_{n}\right)<n$; let $P^{\prime}$ be the permutomino of $\mathcal{C}_{n-1}$ obtained by removing the column $c_{n}$ and the row $r_{\ell\left(c_{n}\right)+1}$ from $P$. Is is then clear that $P$ is produced through the application of operation $(\beta)$ to the $\ell\left(c_{n}\right)$-th cell (from bottom to top) of $P^{\prime}$ (see Figure 8);

3. the rightmost reentrant point of $P$ is of type $\gamma$, and then necessarily $1 \leq \ell\left(c_{n}\right)<n$; let $P^{\prime}$ be the permutomino of $\mathcal{C}_{n-1}$ obtained by removing the column $c_{n}$ and the row $r_{n-\ell\left(c_{n}\right)}$ from $P$. It is then clear that $P$ is produced through the application of operation $(\gamma)$ to the $\left(n-\ell\left(c_{n}\right)\right)$-th cell (from bottom to top) of $P^{\prime}$ (see Figure 9 );

4. the rightmost reentrant point of $P$ is of type $\delta$, also with $\ell\left(c_{n}\right)=n$; due to the permutomino definition, it is clear that $\ell\left(r_{1}\right)=1$, then $P$ has been produced through the application of operation $(\delta)$ to the permutomino $P^{\prime} \in \mathcal{C}_{n-1}$, obtained removing column $c_{n}$ from $P$ (see Figure 10).

The growth of convex permutominoes defined by the ECO operator $\vartheta$ can be suitably represented in terms of the succession rule $\Omega$ :

$$
\Omega:\left\{\begin{array}{l}
(1)_{b} \\
(k)_{b} \rightsquigarrow(1)_{r}(1)_{r} \ldots(k)_{r}(k)_{r}(k+1)_{b}(k+1)_{b} \\
(k)_{r} \rightsquigarrow(1)_{r}(1)_{g} \ldots(k)_{r}(k)_{g}(k+1)_{r} \\
(k)_{g} \rightsquigarrow(1)_{g}(1)_{g} \ldots(k)_{g}(k)_{g} .
\end{array}\right.
$$

The root of the tree is $(1)_{b}$, which is the label of the one-cell polyomino. 


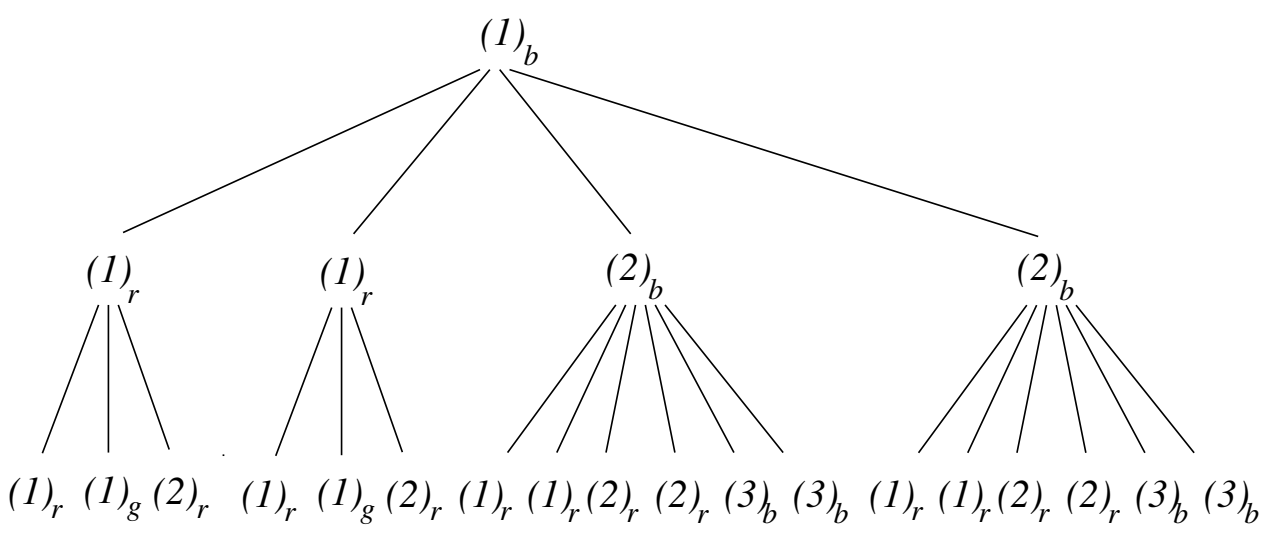

Figure 13: The first three levels of the generating tree of the rule $\Omega$.

\section{Enumeration of convex permutominoes}

In this section we will determine the generating function of convex permutominoes according to various parameters, using the simple remark that the number $f_{n}$ of convex permutominoes of size $n$ is given by the number of objects at level $n$ of the generating tree of $\Omega, n \geq 1$, assuming without loss of generality that the root of the tree is at level 1 .

Let $F$ denote the set of all convex permutominoes. Moreover, for any convex permutomino $P$, let $d(P)$ (briefly, $d$ ) be the degree of $P$, and $n(P)$ (briefly, $n$ ) be the size of $P$. Our aim is to determine the generating function:

$$
F(s, t)=\sum_{P \in F} s^{d(P)} t^{n(P)}=s t+\left(2 s+2 s^{2}\right) t^{2}+\left(8 s+6 s^{2}+4 s^{3}\right) t^{3}+\ldots,
$$

being in particular $F(1, t)$ the generating function of convex permutominoes according to the size. To do this we need to consider the following auxiliary generating functions:

- $B(s, t)=\sum_{P \in B} s^{d(P)} t^{n(P)}$, i.e. the generating function of $B$,

- $R(s, t)=\sum_{P \in R} s^{d(P)} t^{n(P)}$, i.e. the generating function of $R$,

- $G(s, t)=\sum_{P \in G} s^{d(P)} t^{n(P)}$, i.e. the generating function of $G$.

Clearly, $F(s, t)=B(s, t)+R(s, t)+G(s, t)$. From the productions of $\Omega$ we obtain the following relations concerning $B(s, t)$ :

$$
B(s, t)=s t+\sum_{P \in B} 2 s^{d+1} t^{n+1}
$$

hence we have that

$$
B(s, t)=\frac{s t}{1-2 s t} \quad B(1, t)=\frac{t}{1-2 t} .
$$


Then we are able to write down the equation for $R(s, t)$ :

$$
\begin{aligned}
R(s, t) & =\sum_{P \in B} 2\left(s t^{n+1}+\ldots+s^{d} t^{n+1}\right)+\sum_{P \in R}\left(s t^{n+1}+\ldots+s^{d+1} t^{n+1}\right) \\
& =2 s t \sum_{P \in B} \frac{1-s^{d}}{1-s} t^{n}+s t \sum_{P \in R} \frac{1-s^{d+1}}{1-s} t^{n} \\
& =\frac{2 s t}{1-s}(B(1, t)-B(s, t))+\frac{s t}{1-s}(R(1, t)-s R(s, t)) .
\end{aligned}
$$

Let $\bar{B}(s, t)=B(1, t)-B(s, t)$; the previous equation can be re-written as:

$$
R(s, t)\left(1+\frac{s^{2} t}{1-s}\right)=\frac{2 s t}{1-s} \bar{B}(s, t)+\frac{s t}{1-s} R(1, t) .
$$

Equation (5) has two unknowns: $R(s, t)$, and $R(1, t)$. Applying the kernel method (as explained in detail in [2]) we look for the value of $s$ for which the factor on the left multiplying $R(s, t)$ is equal to zero, i.e. the solution of the kernel

$$
1-s+t s^{2}=0
$$

Of the two solutions we observe that only

$$
s_{0}=\frac{1-\sqrt{1-4 t}}{2 t}
$$

is a formal power series with positive coefficients. Substituting $s=s_{0}$ in (5) we have:

$$
\frac{2 s_{0} t}{1-s_{0}} \bar{B}\left(s_{0}, t\right)+\frac{s_{0} t}{1-s_{0}} R(1, t)=0
$$

which leads to:

$$
R(1, t)=\frac{1}{\sqrt{1-4 t}}-\frac{1}{1-2 t} .
$$

Replacing the value of $R(1, t)$ into (5) we obtain

$$
R(s, t)=\frac{s t}{1-s+s^{2} t}\left(\frac{1}{\sqrt{1-4 t}}-\frac{1}{1-2 s t}\right) .
$$

Finally, from the productions of $\Omega$ we derive the following equation for $G(s, t)$ :

$$
\begin{aligned}
G(s, t) & =\sum_{P \in R}\left(s t^{n+1}+\ldots+s^{d} t^{n+1}\right)+\sum_{P \in N} 2\left(s t^{n+1}+\ldots+s^{d} t^{n+1}\right) \\
& =s t \sum_{P \in R} \frac{1-s^{d}}{1-s} t^{n}+2 s t \sum_{P \in N} \frac{1-s^{d}}{1-s} t^{n} \\
& =\frac{s t}{1-s}(R(1, t)-R(s, t))+\frac{2 s t}{1-s}(G(1, t)-G(s, t)) .
\end{aligned}
$$


Again, we set $\bar{R}(s, t)=R(1, t)-R(s, t)$; the previous equation can be re-written as:

$$
G(s, t)\left(1+\frac{2 s t}{1-s}\right)=\frac{s t}{1-s} \bar{R}(s, t)+\frac{2 s t}{1-s} G(1, t) .
$$

As for equation (5), also (6) can be solved using the kernel method. Here the kernel has a unique solution:

$$
s_{1}=\frac{1}{1-2 t}
$$

replacing $s$ with $s_{1}$ in (6) we have:

$$
\frac{s_{1} t}{1-s_{1}} \bar{R}\left(s_{1}, t\right)+\frac{2 s_{1} t}{1-s_{1}} G(1, t)=0
$$

which leads to:

$$
G(1, t)=\frac{1}{2(1-2 t)}+\frac{(1-2 t)^{2}}{2(1-4 t)^{2}}-\frac{1-3 t}{(1-4 t)^{3 / 2}} .
$$

Replacing the expression of $G(1, t)$ in $(6)$ we can obtain also $G(s, t)$,

$$
G(s, t)=\frac{s t}{1-s+s^{2} t}\left[\frac{(s+2) t-1}{(1-4 t)^{3 / 2}}-\frac{4 s^{2} t^{3}-2\left(s^{2}+4 s+2\right) t^{2}+(3 s+4) t-1}{(1-2 s t)(1-4 t)^{2}}\right] .
$$

Finally we have the generating function:

$$
F(s, t)=\frac{s t\left(12 s^{2} t^{3}-2\left(3 s^{2}+4 s-2\right) t^{2}+\left(s^{2}+5 s-4\right) t+1-s\right)}{(1-2 t s)\left(1-s+t s^{2}\right)(1-4 t)^{2}}+\frac{s t^{2}(s-2)}{(1-4 t)^{3 / 2}\left(t s^{2}-s+1\right)},
$$

and the generating function of convex permutominoes according to the size:

$$
F(1, t)=\frac{2 t(1-3 t)}{(1-4 t)^{2}}-\frac{t}{(1-4 t)^{3 / 2}}
$$

Starting from (9) and performing standard calculations we have the following closed form for the number $f_{n}$ of convex permutominoes of size $n$ :

$$
f_{n}=2(n+3) 4^{n-2}-\frac{n}{2}\left(\begin{array}{c}
2 n \\
n
\end{array}\right) \quad n \geq 1
$$

The first terms of the sequence are

$$
1,4,18,84,394,1836,8468, \ldots
$$

We remark that while both the left and the right summands of (10) were in [18] (sequence A079028 and A002457, respectively), the sequence $\left\{f_{n}\right\}_{n \geq 0}$ was not present in the Sloane database before this work (now it appears as sequence A126020). Using the Stirling approximation formula we have that $f_{n} \sim n 4^{n}$. 
Finally we observe that also the number of stack and directed convex permutominoes can be easily obtained from the previous computation.

In fact a stack permutomino can be uniquely represented by a permutomino in class $B$ having the same size. Hence the generating function of stack permutominoes is given by $B(1, t)$, and then the number of stack permutominoes of size $n$, as already stated in [15], is equal to $2^{n}$.

Similarly, a directed convex permutomino can be uniquely represented by a permutomino in class $B$ or one in class $R$ satisfying $\mathbf{U 1}$, having the same size. Hence the generating function of directed convex permutominoes is given by

$$
B(1, t)+\frac{1}{2} R(1, t)=\frac{t}{1-2 t}+\frac{1}{2} \frac{1}{\sqrt{1-4 t}}-\frac{1}{1-2 t}=\frac{1-\sqrt{1-4 t}}{2 \sqrt{1-4 t}},
$$

and then the number of directed convex permutominoes of size $n$, as already stated in $[15]$, is equal to

$$
\frac{1}{2}\left(\begin{array}{c}
2 n \\
n
\end{array}\right)
$$

\section{Further work}

In this paper we solve the problem of determining a closed formula for the number of convex permutominoes with a fixed size. We reach this goal by defining a recursive construction of all the permutominoes of size $n+1$ starting from those of size $n$, for any $n \geq 1$.

Several problems on the class of permutominoes however remain still open. Below we propose a small list of the problems which we are interested in, and we would like to tackle in some future work:

1. to give a combinatorial interpretation of the closed formula (10) for the number of convex permutominoes. We remark that the expression of $f_{n}$ resembles the expression (1) for the number $c_{n}$ of convex polyominoes of semi-perimeter equal to $n+2$; in particular it is easy to check that the numbers $c_{n}$ and $f_{n}$ are related by the following simple relation:

$$
c_{n+2}=f_{n}+\left(\begin{array}{c}
2(n-1) \\
n-1
\end{array}\right)+4^{n-2} \quad n \geq 2,
$$

which requires a combinatorial proof.

2. we believe that the ECO construction of convex permutominoes can be extended to the class of column-convex permutominoes. One of the problems with this class is that here Proposition 2 does not hold as shown in Figure 14.

3. enumerate convex permutominoes according to the area, i.e. the number of cells of the permutomino. The ECO construction we have determined easily leads to a functional equation satisfied by the generating function of convex permutominoes according to the area and the size of the permutomino; however, then we have not been able to solve this equation. 


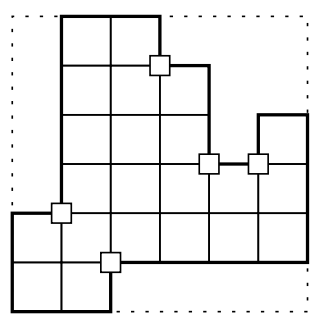

Figure 14: A column-convex permutomino: its reentrant points do not satisfy the statement of Proposition 2.

\section{References}

[1] Bacchelli, S., Barcucci, E., Grazzini, E., Pergola, E.: Exhaustive generation of combinatorial objects by ECO, Acta Informatica 40 (2004) 585-602.

[2] Banderier, C., Bousquet-Mélou, M., Denise, A., Flajolet, P., Gardy, D., GouyouBeauchamps, D.: Generating functions for generating trees, Disc. Math. 246 (2002) 29-55.

[3] Barcucci, E., Del Lungo, A., Pergola, E., Pinzani, R.: ECO: a methodology for the Enumeration of Combinatorial Objects., J. Diff. Eq. and App. 5 (1999) 435-490.

[4] Barcucci, E., Frosini, A., Rinaldi, S.: On directed-convex polyominoes in a rectangle, Disc. Math., 298 (2005) 62-78.

[5] Boldi, P., Lonati, V., Radicioni, R., Santini, M.: The number of convex permutominoes., Rapporto Interno n.311-06, Università degli Studi di Milano, available electronically at http://santini.dsi.unimi.it/research/papers.php.

[6] Bousquet-Mèlou, M., A method for the enumeration of various classes of columnconvex polygons, Disc. Math. 154 (1996) 1-25.

[7] Bousquet-Mèlou, M., Guttmann, A. J., Enumeration of three dimensional convex polygons, Ann. of Comb. 1 (1997) 27-53.

[8] Brak, R., Guttmann, A. J., Enting, I. G.: Exact solution of the row-convex polygon perimeter generating function, J. Phys. A 23 (1990) L2319-L2326.

[9] Brlek, S., Labelle, G., Lacasse, A.: A Note on a Result of Daurat and Nivat, Lecture Notes in Computer Science, Springer Berlin/Heidelberg, Vol. 3572 (2005) 189-198.

[10] Chang, S.J., Lin, K.Y.: Rigorous results for the number of convex polygons on the square and honeycomb lattices, J. Phys. A: Math. Gen. 21 (1988) 2635-2642.

[11] Conway, J.H., Lagarias, J.C.: Tiling with polyominoes and combinatorial group theory, J. Comb. Th. A 53 (1990) 183-208.

[12] Daurat, A., Nivat, M.: Salient and reentrant points of discrete sets, Disc. Appl. Math. 151 (2005) 106-121. 
[13] Delest, M., Viennot, X.G.: Algebraic languages and polyominoes enumeration, Theor. Comp. Sci. 34 (1984) 169-206.

[14] Del Lungo, A., Duchi, E., Frosini, A., Rinaldi, S.: On the generation and enumeration of some classes of convex polyominoes, El. J. Combinatorics, 11 (2004), \#R60.

[15] Fanti, I., Frosini, A., Grazzini, E., Pinzani, R., Rinaldi, S.: Polyominoes determined by permutations, Proceedings of MathInfo 06, Fourth Colloquium on Mathematics and Computer Science Algorithms, Trees, Combinatorics and Probabilities September 18-22 2006, Nancy, Disc. Math. Theor. Comp. Sci. AG 381-390 (2006).

[16] Ferrari, L., Pergola, E., Pinzani, R., Rinaldi, S.: An algebraic characterization of the set of succession rules, Theor. Comp. Sci., 281 (1-2) (2002) 351-367.

[17] Incitti, F., Permutation diagrams, fixed points and Kazhdan-Lusztig $R$-polynomials, Ann. Comb., 10, N.3, (2006) 369-387.

[18] Sloane, N.J.A.; The On-Line Encyclopedia of Integer Sequences, http://www.research.att.com/ njas/sequences/ 